\title{
Stochastic optimization for the daily joint operation of wind/PV and energy storage
}

\author{
I.L.R. Gomes ${ }^{1}$, H.M.I. Pousinho ${ }^{2}$, R. Melício ${ }^{1,2}$, V.M.F. Mendes ${ }^{1,3}$ \\ ${ }^{1}$ Departamento de Física, Escola de Ciências e Tecnologia, Universidade de Évora, Portugal \\ ruimelicio@gmail.com \\ ${ }^{2}$ IDMEC, Instituto Superior Técnico, Universidade de Lisboa, Lisbon, Portugal \\ ${ }^{3}$ Instituto Superior of Engenharia de Lisboa, Lisbon, Portugal
}

\begin{abstract}
This paper deals with the problem of optimal bidding in a day-ahead market of electricity for a power producer having joint operation of wind with photovoltaic power systems and storage of energy. Uncertainty, not only on electricity market prices, but also on wind and photovoltaic powers, has to be faced in order to achieve optimal bidding. The problem is viewed as a sort of a two-stage stochastic optimization problem formulated by mix-integer linear programming. A case study with data from the Iberian Peninsula is presented and a comparison between joint and disjoint operations is discussed, allowing concluding that the joint operation attenuates the economic impact of disjoint operation volatility.
\end{abstract}

Keywords: Day-ahead electricity market; energy storage; mixed-integer linear programming; stochastic optimization; wind with PV powers.

\section{Introduction}

Although of the uncertainty on the availability of power, renewable energy sources (RES) exploitations are the main announced measures to reduce anthropogenic greenhouse gases emissions and the way of future in what regards a sustainable development [1]. The exploitation of RES has worldwide grown rapidly, benefiting from sustenance schemes and incentive policies [2]. A main characteristic of the RES exploitation of wind power (WP) and of photovoltaic power (PVP) is uncertainty, which have to be considered in the scheduling of these powers. WP or PVP systems standing alone are not capable of warranting power generation during all day due to the uncertainty in the availability of the source of energy at different time periods of day or different periods of the year. The mismatch between periods of low availability of these sources lead to a question about if complementarity between WP and PVP has a better appropriateness for presenting a single offer in a day-ahead market (DAM). Also, energy storage is a way to harness RES, allowing the store and discharge of energy at adequate conditions. A report of the NREL states that the challenges associated with meeting the variation in demand while providing reliable services has motivated historical development of the storage of energy and that large penetrations of variable generation rises the need for flexibility options [3]. Large scale RES integration without energy storage is said to be a challenge for future power systems [4]. Hence, storage technologies play an important role in the actual deregulated markets, providing capability for arbitrage, increasing the economic value of RES [5]. Joint operation with the aim of submitting in a DAM a single bid of a producer having WP, PVP and a battery for energy storage is the line of research perused in this paper. Which has as a contribution the application of MILP approaches to two-stage stochastic 
optimization problems for optimal bid of: WP; PVP and joint operation of WP, PVP and energy storage device.

\section{Relationship to Smart Systems}

Intelligence in the form of algorithms, new computing models, and advanced analytics prone to allows enhancement in decision making [6]. Power system can benefit from this intelligence. A smart system can be stated as an embedded system that incorporates advanced systems and provide the inhabitants with sophisticated monitoring and control over how something happens in the system [7], for example, the management of a wind system, a PV system or a storage device. Smart systems are capable of sensing, making diagnosis, describing, qualifying and managing how something happens in the system, incorporating both technical intelligence and cognitive functions. This incorporation is labeled as the third-generation of smart systems, offering an interface between the virtual and the physical world. Smart systems are highly reliable, often miniaturized, networked, predictive and energy autonomous [8]. The exponential increase in energy demand, that is forecasted to be around $45 \%$ by 2030 , and the increase integration of RES in power systems are facts driving research and development about managing how things happen in the system [9]. Particularly, energy management will be one of the most urgent topics of the century and a significant driver for research and developments [9]. Important issues in energy management are reliability and efficiency. These issues are implied by the change of paradigm from the traditional electric grid to a smart grid one [9]. A smart grid in power system can lead to better results in decision-making under uncertainty. Particularly, monitoring and high quality real-time data for the exploitation of WP or PVP are a valuable contribution to mitigate the implications of uncertainty, mitigating the loss of a producer's profit due to bad decisions of bidding in a day-ahead electricity market. So, smart systems in an electric grid are expected to be: crucial for increase of power reliability; favorable for achievement of better bidding in electricity markets and an overall enhancement in power system.

\section{State of the Art}

One of the greatest challenges for exploitation of WP or PVP is to face the intermittence and variability of those powers in order to mitigate the uncertainty in power generation. Exploitation of WP or PVP by a power producer bidding in DAM at day d-1 has a menace. Which is due to the power output delivered at day $\mathrm{d}$ being predisposed to differ from the clearing accepted level of power in the market. Consequently, the power producer enters in imbalance of delivering and is subjected to economic penalty. Therefore, uncertainty in power output has to be properly accommodated into the decision of bidding. A complementarity of WP with PVP has been shown on the Iberian Peninsula, leading to a question about if the joint exploitation of the operation of WP with PVP is able to mitigate the uncertainty in the power output [10]. Literature dealing with WP producer (WPP) mitigation of WP uncertainty favors the implementation of combining strategy with the aim of reducing uncertainty on power generation. This 
combining strategy for a WPP includes the use of energy storage technology, namely pump-storage plants, compressed air facilities or vanadium redox flow batteries [11-13]. Also, the use of: purchase call/put options to pumped-storage facility is proposed for wind producers to hedge against WP uncertainty [14]; joint operation of WP, PVP, pump-storage power plant and energy storage devices is proposed for reserve market [15].

Stochastic nonlinear programming is an approach suitable for the bidding strategy of a WPP to achieve convenient decision with the aim of considering uncertainty [16]. Even more suitable, if an approach supported by MILP is conceivable. MILP either deterministic [17] or stochastic [18] have been proposed to convenient address the appropriated decision of bidding strategy a WPP needing to consider uncertainty.

\section{Profit Description}

A power producer exploiting WP or PVP needs to consider the uncertainty on the availability of power impacts in the worth of bidding in a DAM. If joint operation of the exploitation of these powers with energy storage is possible is important to further research the worth of profiting from the mismatch between periods of low availability of these powers. This research is the subject of the following problem description, having the objective of enquiring if this joint operation can improve profit of the power producer in DAM. A prerequisite is that the market rules permit that joint operation participates in the aforementioned electricity market. The concept presented in this paper is the same of the concept of a Virtual Power Plant (VPP). A VPP is defined as a power plant that aggregates different distributed generation units, such as WP and PVP systems, in order to participate in the market as a single entity. This participation is expected to not only facilitate trading of energy in electricity markets, but also being prone to achieve better profits.

The power system balance between the total amount of energy converted into electric energy and the usage of electric energy has to be null in real time. This balance has to be guaranteed with a convenient anticipation relatively to the real time delivering. Usually, the balance is guaranteed by production/consumption programs established in advance that guarantees system balance and by a real-time balancing market. The producer imbalance or the system imbalance may be negative, null or positive in the market, but as long as there is producer imbalance the producer is subjected to a market penalty procedure. The procedure in the Iberian electricity market is to subject the producer to a price for the positive energy imbalance and another price for negative energy imbalance. In the Iberian electricity market the procedures for computing these prices are reported in [19]. The imbalance incurred by a power producer with a power plant $k$ in a disjoint assessment in time $t$ is stated as follows:

$$
d_{t}^{k}=P_{t s}^{k}-P_{t}^{k} .
$$

In (1) $P_{t s}^{k}$ is the physical delivering of energy in time $t$ and $P_{t}^{k}$ is the bid submitted in the DAM. An imbalanced of a physical energy delivering is associated with a price ratio: positive and negative imbalances are associated with price ratios $\mathrm{pr}_{t}^{+}$and $\mathrm{pr}_{t}^{-}$, respectively. The positive price ratio is stated as follows: 


$$
p r_{t}^{+}=\frac{\lambda_{t}^{+}}{\lambda_{t}}, p r_{t}^{+} \leq 1 .
$$

The negative price ratio is defined as follows:

$$
p r_{t}^{-}=\frac{\lambda_{t}^{-}}{\lambda_{t}}, p r_{t}^{-} \geq 1
$$

In (2) and (3) $\lambda_{t}$ is the DAM price and $\lambda_{t}^{+}$and $\lambda_{t}^{-}$are respectively the positive and negative imbalance prices. Consequently, the power plant $k$ at period $t$ in a disjoint assessment has a profit given as follows:

$$
P R_{t}^{k}=\lambda_{t} P_{t}^{k}+\left(\lambda_{t} p r_{t}^{+} d_{t}^{k+}-\lambda_{t} p r_{t}^{-} d_{t}^{k-}\right)
$$

In (4) the first term represents the profit associated with the DAM price $\lambda_{t}$ from an accepted production $P_{t}^{k}$. The term in brackets is associated with the income derived from the procedure of economic penalty. The positive and negative deviations are respectively quantified by $d_{t}^{k+}$ and $d_{t}^{k-}$ subjected to the constraint of at least one of the deviations is always null at a period $t$.

\section{Problem Formulation}

The goal of the optimization problem is the profit maximization. Maximization of profit implies a convenient management of the economic impact of energy delivering deviations. This management has to face uncertainty on market prices and on availability of energy in due time to be converted into electric energy in order to satisfy delivering. If there is a mismatch between the energy traded and the physical delivering, then eventual losses on profit can occur. The uncertainties on WP and on PVP availabilities are modelled by a convenient selection of scenarios from a set $S$ said to be the set of appropriate scenarios for the next day. A scenario $s$ has the probability $\pi_{s}$. The MILP formulation for the disjoint operation of WP or PVP systems are equivalent problems stated as follows:

$$
\max \sum_{s=1}^{S} \sum_{t=1}^{T} \pi_{s}\left(\lambda_{t s} P_{t}^{k}+\lambda_{t s} p r_{t s}^{+} d_{t s}^{k+}-\lambda_{t s} p r_{t s}^{-} d_{t s}^{k-}\right)
$$

Energy offer constraints

$$
0 \leq P_{t}^{k} \leq P^{k \max }, \forall t
$$

Imbalance constraints

$$
\begin{gathered}
d_{t s}^{k}=P_{t s}^{k}-P_{t}^{k}, \forall t, \forall s \\
d_{t s}^{k}=d_{t s}^{k+}-d_{t s}^{k-}, \forall t, \forall s \\
0 \leq d_{t s}^{k+} \leq P_{t s}^{k} u_{t s}^{k}, \forall t, \forall s \\
0 \leq d_{t s}^{k-} \leq P^{k \max }\left(1-u_{t s}^{k}\right), \forall t, \forall s .
\end{gathered}
$$

In (6) the limit on the bid is set to be the rated power of the $k$ unit (wind or PV system).

In (7) to (10) the imbalance is decomposed into a difference of positive values, i.e., the 
difference between the positive $d_{t s}^{k+}$ and the negative $d_{t s}^{k-}$ imbalances. If the imbalance is negative, the term $\lambda_{t s} p r_{t s}^{+} d_{t s}^{k+}$ is null and the term $\lambda_{t s} p r_{t s}^{-} d_{t s}^{k-}$ is subtracted. If the imbalance is positive the term $\lambda_{t s} p r_{t s}^{-} d_{t s}^{k-}$ is null and the term $\lambda_{t s} p r_{t s}^{-} d_{t s}^{k-}$ is added. The problem for joint operation of WP, PVP and energy storage is formulated as a sort of a two-stage stochastic optimization problem where the hourly bids and imbalances are first and second-stage variables, respectively. The goal of the problem is to find a single optimal bid in DAM for the wind power, PVP generation and energy storage. The stochastic MILP formulation is specified by the maximization problem stated as follows:

$$
\max \sum_{s=1}^{S} \sum_{t=1}^{T} \pi_{s}\left(\lambda_{t s} P_{t}+\lambda_{t s} p r_{t s}^{+} d_{t s}^{+}-\lambda_{t s} p r_{t s}^{-} d_{t s}^{-}\right) .
$$

General constraints

a) Energy offer constraint

$$
0 \leq P_{t} \leq P^{W \max }+P^{P V \max }+P_{t}^{D e \max } .
$$

b) Output power of combined wind power, $P V$ power and energy storage device

$$
P_{t s}=P_{t s}^{W}+P_{t s}^{P V}-P_{t}^{\text {Chbat }}+P_{t}^{\text {Debat }} .
$$

Imbalance constraints

$$
\begin{gathered}
d_{t s}=P_{t s}-P_{t}, \forall t, \forall s \\
d_{t s}=d_{t s}^{+}-d_{t s}^{-}, \forall t, \forall s \\
0 \leq d_{t s}^{+} \leq P_{t s} u_{t s}, \forall t, \forall s \\
0 \leq d_{t s}^{-} \leq\left(P^{P V \max }+P^{P V \max }+P^{D e \max }\right)\left(1-u_{t s}\right), \forall t, \forall s .
\end{gathered}
$$

Constraints of energy storage device

a) Energy storage equation

$$
E_{t}^{\text {bat }}=E_{t-1}^{\text {bat }}+\eta^{\text {Chbat }} P_{t}^{\text {Chbat }}-\frac{1}{\eta^{\text {Debat }}} P_{t}^{\text {Debat }} .
$$

b) Energy storage limits

$$
0 \leq E_{t}^{\text {bat }} \leq E^{\text {bat max }} .
$$

c) Storage power limits

$$
\begin{gathered}
0 \leq P_{t}^{\text {Chbat }} \leq P_{t}^{\text {Chbat } \max } k_{t} . \\
0 \leq P_{t}^{\text {Debat }} \leq P_{t}^{\text {Debat max }}\left(1-k_{t}\right) .
\end{gathered}
$$

In (18) is the equation for the model of a Vanadium redox flow battery, assuming a null depth of discharge and considering the efficiency of charging and of discharging. This type of energy storage is one of the most promising technology for energy management in order to alleviate the variation and intermittence of WP or PVP.

The procedure of the joint bid strategy is shown in Fig. 1. 


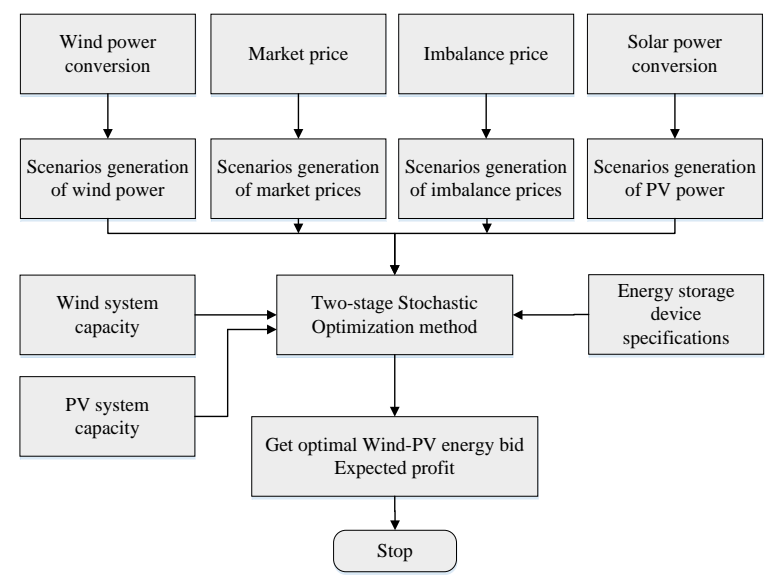

Fig 1. Flowchart of joint bidding strategy.

In Fig. 1 the upper blocks are for scenario generation procedures obtained via historical data of wind speed, solar irradiance and historical data of the Iberian electricity market. After the blocks for scenario generation procedures, technical data blocks access the wind system, PV system capacities and energy storage speciation to deliver the information into the block implementing the MILP approach for the two-sage stochastic optimization method in GAMS/CPLEX.

\section{Case Study}

The case study is based on a wind-PV system in the Iberian Peninsula with rated power of $200 \mathrm{MW}$ and an energy storage rated power of $30 \mathrm{MW}$. The day-ahead energy prices and the price ratios $p r_{t}^{+}$e $p r_{t}^{-}$are reported in [20]. The bidding is for a $24 \mathrm{~h}$ horizon on an hourly basis. The scenarios are 10 for WP, 10 for PVP, 10 for DAM prices and 10 for price ratios. The DAM-clearing price scenarios and the price ratios scenarios $p r_{t}^{+}$and $p r_{t}^{-}$are shown in Fig. 2.
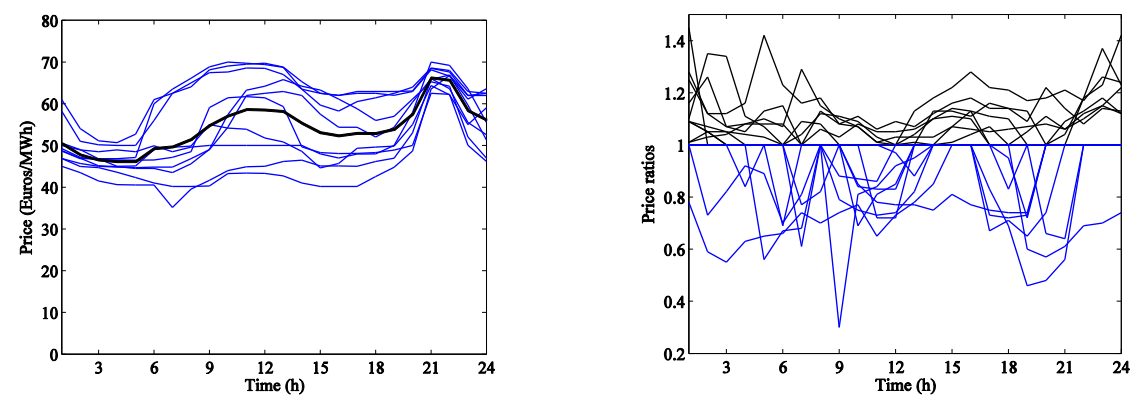

Fig 2. Left, electricity price (average - black line). Rigth, price ratios: positive (blue lines), negative (black lines).

The scenarios for WP and for PVP are shown in Fig. 3. 

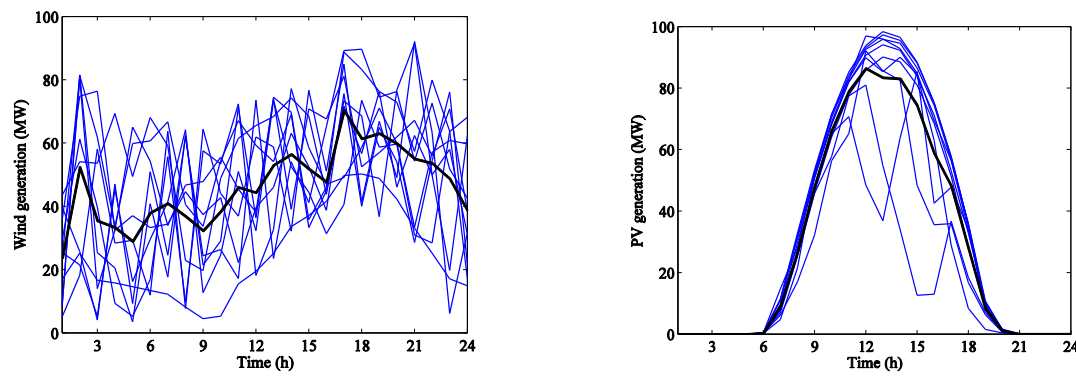

Fig 3. Left, WP scenarios and average scenario (black line). Rigth, PV scenarios and average scenario (black line).

The energy traded and the absolute value of the energy deviation by the disjoint and by the joint operations are shown in Fig. 4.
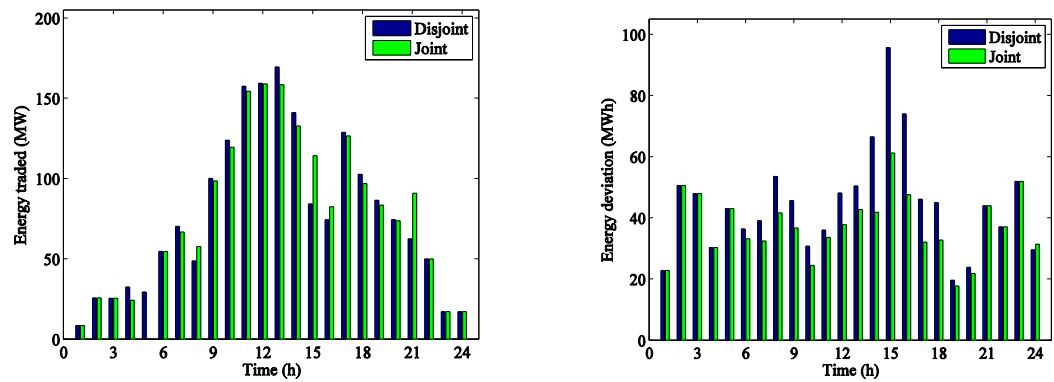

Fig 4. Left: energy traded. Right: absolute energy deviation.

In Fig.4, absolute energy deviation is the sum of the positive with the negative imbalances. Energy is stored at $5 \mathrm{~h}$ and delivered at the favorable price hour $21 \mathrm{~h}$. The expected profits for the disjoint and joint with the energy storage operations are $98308.96 €$ and $98608.98 €$, respectively. Hence, an increase of $300 € /$ day is expected with this joint operation. A higher increase occurs at $15 \mathrm{~h}$, a period of favorable prices, low negative imbalance price ratios and high positive imbalance price ratios. Although, the numeric values change in function of availability of WP and PVP, the energy deviation is conveniently managed in what regards economic penalties in order to achieve better profit, i.e., this joint operation is never worse than the disjoint one.

\section{Conclusion}

In this paper, an optimization problem of bidding in DAM for a power producer having joint operation of WP with PVP and energy storage of the type Vanadium redox flow battery is presented. The problem is stated as sort of a two-stage stochastic optimization problem addressed as MILP problem. The joint bidding is envisaged as a favorable one when the mismatch of uncertainty due to the WP and the PVP is partial disabled by one another and an energy storage device allows flexibility of storing energy and discharging at hours of convenient DAM prices. A future work can include the participation in intraday-markets. 


\section{Acknowledgments}

To thank the Millennium BCP Foundation for the financial support; and FCT, through IDMEC, under LAETA, ref. UID/EMS/50022/2013.

\section{References}

1. Gutierrez, M.F., Silva, R.A., Montoro, P.P.: Effects of wind intermittency on reduction of $\mathrm{CO}_{2}$ emissions: the case of the Spanish power system. Energy 61, 108-117 (2013)

2. Wang, T., Gong, Y., Jiang, C.: A review on promoting share of renewable energy by greentrading mechanisms in power system. Renewable and Sustainable Energy Reviews 40, 923-929 (2014)

3. Denholm, P., Ela, E., Kirby, B., Milligan, M.: The role of energy storage with renewable electricity generation. National Renewable Energy Laboratory, Colorado, USA (2010)

4. Beaudin, M., Zareipour, H., Schellenberglabe, A., Rosehart, W.: Energy storage for mitigating the variability of renewable electricity sources: an updated review. Energy for Sustainable Development 14(4), 302-314 (2010)

5. Divya, K.C., Østergaard, J.: Battery energy storage technology for power systems-an overview. Electric Power Systems Research 79(4), 511-520 (2009)

6. Gomes, I.L.R, Pousinho, H.M.I, Melício, R., Mendes, V.M.F.: Bidding and optimization strategies for wind-pv systems in electricity markets assisted by CPS. Energy Procedia 106, $111-121(2016)$

7. Arsan, T.: Smart systems: from design to implementation of embedded smart systems. In: 13th International Symposium on Smart MicroGrids for Sustainable Energy Sources enabled by Photonics and IoT Sensors, Nicosia, Cyprus, 59-64 (2016)

8. Topham, D., Gouze, N.: Smart systems integration knowledge gateway. In: European Technology Platform on Smart Systems Integration, Turin, Italy (2015)

9. Glesner, M., Philip, F.: Embedded systems design for smart system integration. In: IEEE Computer Society Annual Symposium on VLSI, Natal, Brazil 32-33 (2013)

10. Jerez, S., Trigo, R.M., Sarsa, A., Lorente-Plazas, R., Pozo-Vásquez, D., Montávez, J.P.: Spatio-temporal complementarity between solar and wind power in Iberian Peninsula. Energy Procedia 40, 48-57 (2013)

11. González-Garcia, J., Muela, R.M.R., Santos, L.M., González, A.M.: Stochastic joint optimization of wind generation and pumped-storage units in an electricity market. IEEE Transaction on Power Systems 23(2), 460-468 (2008)

12. Angarita, J.L., Usaola, J., Martínez-Crespo, J.: Combined hydro-wind generation bids in a pool-based electricity market. Electric Power Systems Research 79(7), 1038-1046 (2009)

13. Sundararagavan, S., Baker, E.: Evaluating energy storage technologies for wind power integration. Solar Energy 86(9), 2707-2717 (2012)

14. Hedman, K.W., Sheble, G.B.: Comparing hedging methods for wind power: using pumped storage hydro units vs. options purchasing. In: Proc. International Conference on Probabilistic Methods Applied to Power Systems - PMAPS, Stockholm, Sweden 1-6 (2006)

15. Parastegari, M., Hooshmand, R.A., Khodabakhshian, A., Zare, A.H.: Joint operation of a wind farm, photovoltaic, pump-storage and energy storage devices in energy and reserve markets. Electrical Power and Energy Systems 64, 275-284 (2015)

16. Matevosyan, J., Söder, L.: Minimization of imbalance cost trading wind power on the shortterm power market. IEEE Transactions on Power Systems 21(3), 1396-1404 (2006)

17. Pousinho, H.M.I., Mendes, V.M.F., Catalão, J.P.S.: Investigation on the development of bidding strategies for a wind farm owner. International Review of Electrical Engineering 5(3), 1324-1329 (2010)

18. Laia, R., Pousinho, H.M.I., Melício, R., Mendes, V.M.F.: Self-scheduling and bidding strategies of thermal units with stochastic emission constraints. Energy Conversion and Management 89, pp. 975-984 (2015)

19. Gomes, I.L.R, Pousinho, H.M.I, Melício, R., Mendes, V.M.F.: Optimal wind bidding strategies in day-ahead markets. In: technological Innovation for Cyber-Physical Systems, Springer 470, pp. 475-484 (2016)

20. http://www.esios.ree.es/web-publica/ 